\title{
Synthesis and Surfactant Studies of Dialkyl Dimethyl Quaternary Ammonium Bromide Formulations
}

\author{
Rocky Barney, Tony Stark, and Dru Delaet* \\ Department of Physical Science \\ Southern Utah University \\ Cedar City, Utah 84720 USA
}

Received: July 15, $2005 \quad$ Accepted: November 22, 2005

\begin{abstract}
Quaternary ammonium salts (otherwise known as quats) are commonly used active ingredients in biocide formulations used in the anti-microbial industry. Although quats have been established to be effective biocides, there are few studies investigating the maximization of biocidal efficacy in multiple component formulations using various carbon chain lengths. Reported here is the synthesis based on the $\mathrm{Sn}^{2}$ reaction of tertiary amines with alkyl bromide. Surfactant studies of the single and dual component systems were conducted, and the evaluation is explored.
\end{abstract}

\section{INTRODUCTION}

Quaternary ammonium compounds (Quats) are an important class of surfactants. They are utilized in a wide variety of applications which include, but are not limited to, fungicides, bleach activators, biocides, algaecides, softeners, and conditioners [1]. There are several different types of quats used as biocides, with the benzalkonium being the most common. Benzalkoniums have been shown to be effective as a biocide, but recent data indicates that various strains of benzalkonium-resistant bacteria have come into being [2, 3]. For these reasons we have chosen to study the surfactant properties of both mono and dialkyl ammonium bromide compounds and their formulations. We hope that this will lead us to a better understanding of the fundamental properties of the quats and their various formulations, which will ultimately lead to the development of improved, bacteria-resistant formulations of quaternary ammonium compounds.

\section{EXPERIMENTAL}

Synthesis of compounds was carried out using two methods; a previously described method absent of solvent [4], and

* Corresponding author the standard "textbook" method for quaternary ammonium halide synthesis [5]. Both methodologies undergo a bimolecular nucleophillic substitution reaction (Sn2). The following general procedure was followed for all syntheses: A tertiary amine and an $\mathrm{N}$-substituted alkyl halide were placed into a $1000 \mathrm{ml}$ 3-neck roundbottom flask in a 1:1 ratio. The amine was always added first followed by addition of the alkylhalide. The solution was heated with stirring to a temperature below the boiling point of the solution, but hot enough to keep the solution molten. The temperature range was between 75 and 180 degrees Celsius. The solution was allowed to react for a minimum of ten hours, at which time there was a pronounced change in both the color and consistency of the solution.

The quats and various formulations of the quats were evaluated using an emulsion stability test. The emulsion stability in this method is subject to multiple interfaces; a liquid-liquid interface, a gasliquid interface, and a solid-liquid interface. $5 \mathrm{ml}$ of a solution having .05 mass percent of surfactant was transferred to a graduated cylinder. This was followed by the addition of $5 \mathrm{ml}$ of decane to the graduated cylinder. Each analysis was recorded in duplicate to eliminate erroneous errors. Each cylinder was agitated once per second for ten seconds. After the ten-second interval, time 


\section{Single Component}

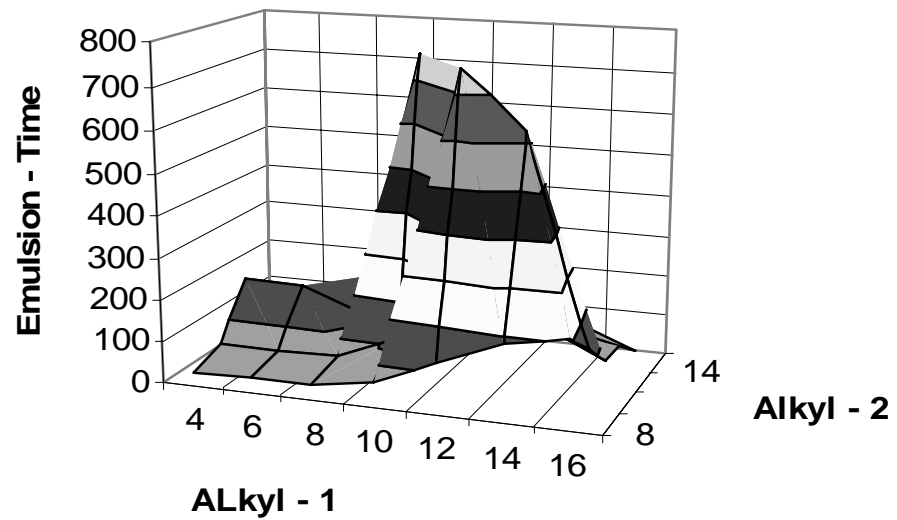

Figure 1. Time required for the initial emulsion to break of a $0.05 \mathrm{wt} \%$ dialkyl dimethyl ammonium bromide solutions.

\section{Single Component}

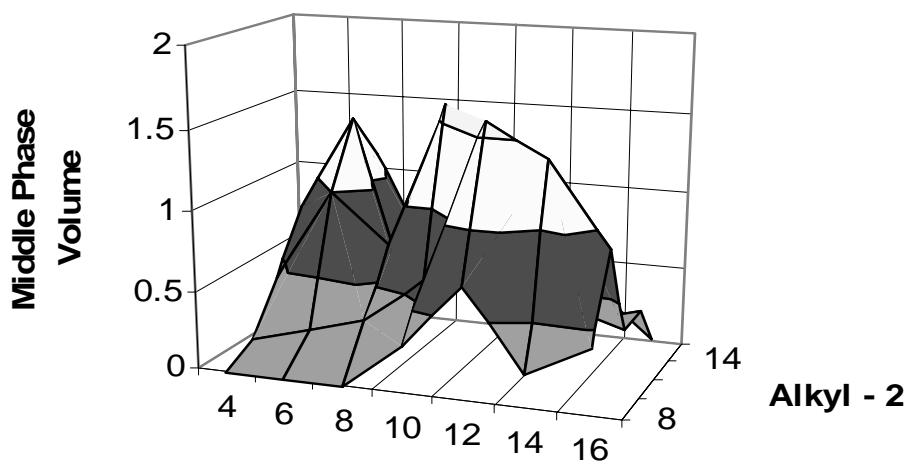

Figure 2. Stable middle phase micro-emulsion volume of a $0.05 \mathrm{wt} \%$ dialkyl dimethyl ammonium bromide solutions.

\section{Surfactant Mixture $(\mathbf{1 0 , 1 0 )}$}

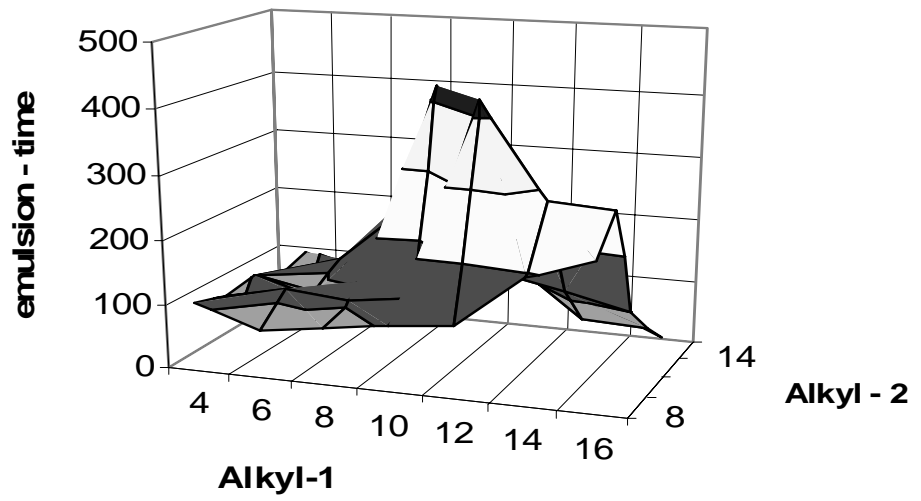

Figure 3. Time required for the initial emulsion to break of a 50/50 mixture of dimethyl didecyl ammonium bromide with another dialkyl dimethyl ammonium bromide all in a $0.05 \mathrm{wt} \%$ solutions. 


\section{Surfactant Mixture $(\mathbf{1 0 , 1 0 )}$}

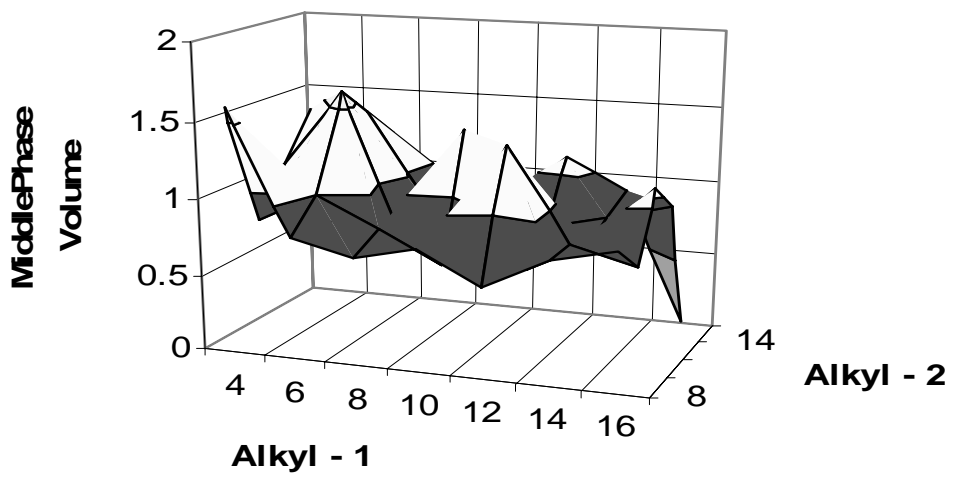

Figure 4. Stable middle phase micro-emulsion volume of a 50/50 mixture of dimethyl didecyl ammonium bromide with another dialkyl dimethyl ammonium bromide all in a $0.05 \mathrm{wt} \%$ solutions.

\section{Didecyl Dimethyl Ammonium Bromide Mixtures with Other Dialkyl Ammonium Compounds}

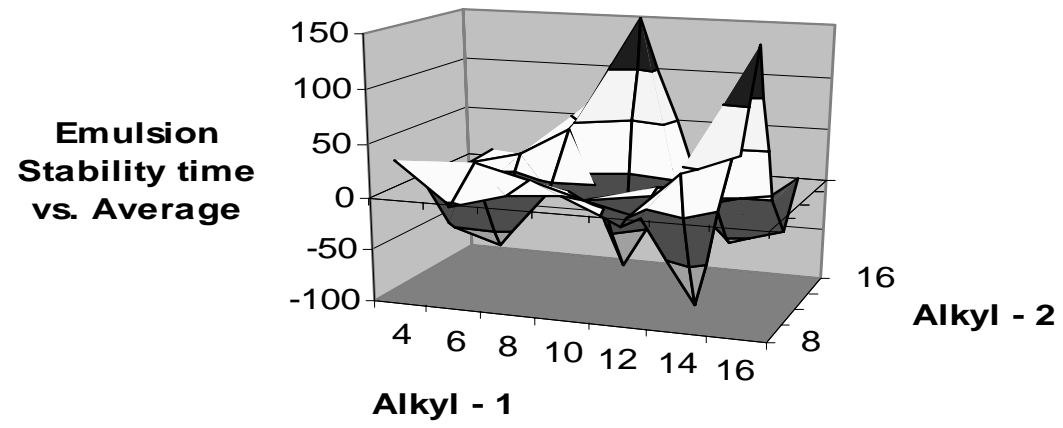

Figure 5. The variation from the mathematical average time required for the initial emulsion to break of the single components and the actual measured value of the mixture.

of phase separation was measured with the use of a stopwatch, and the volume of emulsion identified. Each analysis was then repeated after dilution. When high surfactant concentrations are present, emulsion volume is maximized. However, a positive relationship exists between the concentration and the phase separation time.

\section{RESULTS AND DISCUSSION}

As expected, some general trends for the single component systems were observed. As alkyl chain length increases, there is a general increase in both emulsion volume and emulsion time. As seen in Figures 1 and 2, both graphs show a marked increase at the 8,14 quat, followed by a slow decrease in both properties. This data is consistent with what was predicted; since the increased carbon length provides a larger nonpolar surface for the Van der Waals interaction with the oil of the emulsion.

The system consisting of 10,10 quat and a quat of variable carbon chain length was investigated. We chose to look at the 10,10 quat because previous data had indicated that it was the most effective biocide of the single component systems. The binary quat system provided data that was far more interesting than that of the single component system. As alkyl chain length increased, so did emulsion time. The data also confirmed the marked increase in emulsion time at the 8,14 quat. This data is consistent with what one would expect (Figure 3). The deviation from our expectation occurs when we investigate the 


\section{Didecyl Dimethyl Ammonium Bromide Mixtures with Other Dialkl Ammonium Compounds}

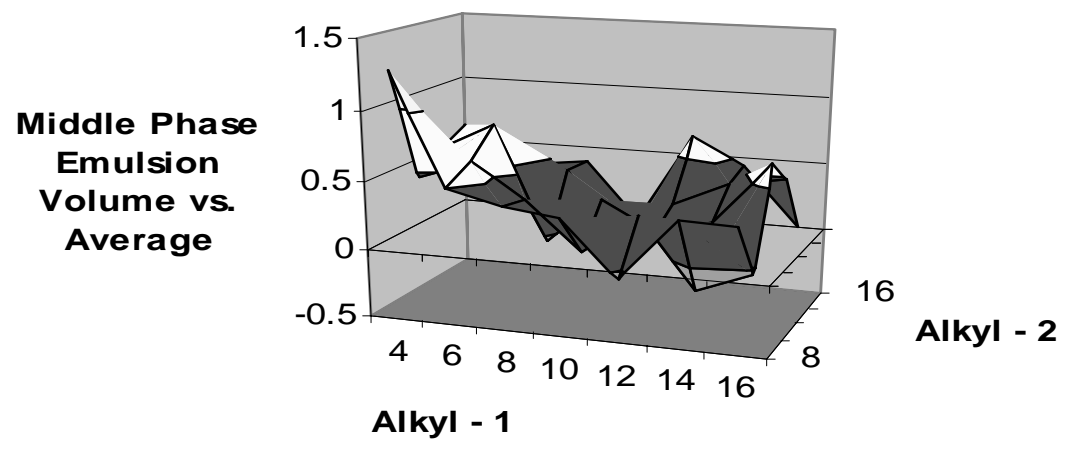

Figure 6. The variation from the mathematical average middle phase emulsion volume for the single components and the actual measured value of the mixture.

\section{Alkyl Decyl Dimethyl Ammonium Bromide - Decane and Cyclohexane Comparison}

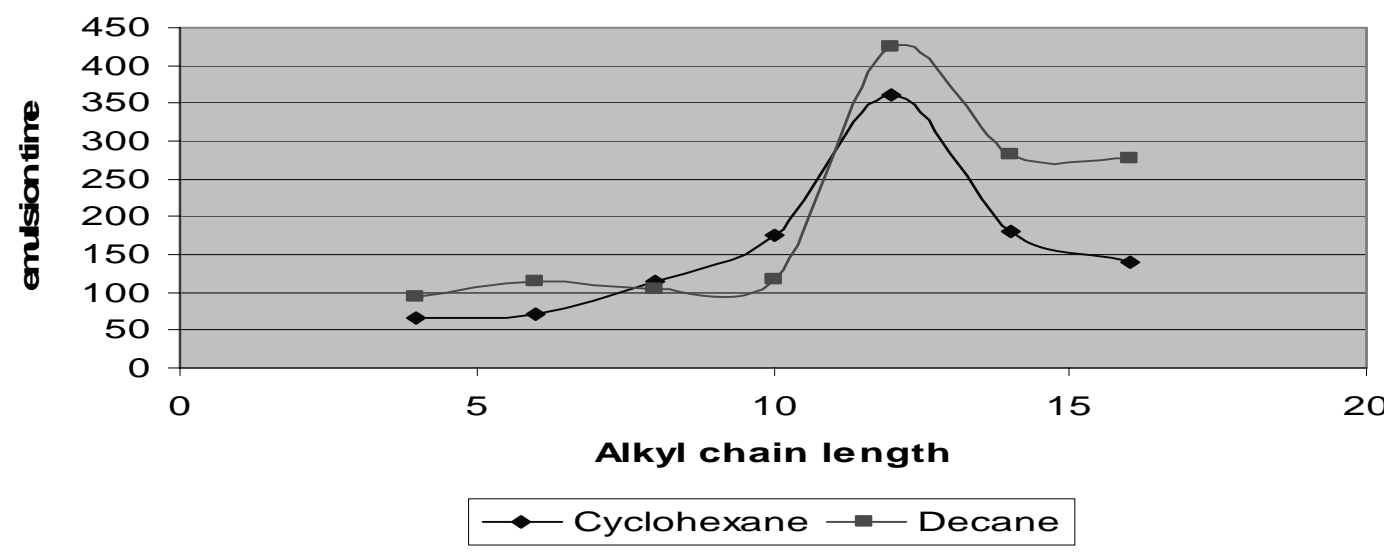

Figure 7. The comparison of decane and hexane as the oil phase in measuring the emulsion time for samples of alkyl decyl dimethyl ammonium bromide solutions at $0.05 w t \%$.

data pertaining to emulsion stability for the binary system. The most notable feature of was the peak at eh 4,14 quat (Figure 4). This interesting phenomena warranted further investigation in to the binary system. Next, we sought to investigate any antagonistic/synergistic relationship that may exist between the two quats. This was accomplished by first establishing a base line. The base line was calculated by computing an average of the effects that the two quats exhibited on the binary system at the concentration which they existed in solution $(.025 \%)$. Next, we plotted the base line average values against the observed values (Figure 5). It was immediately apparent that there is both synergistic and antagonistic character within the binary system. To further explore and evaluate this characteristic, we plotted the amount of deviation from the expected value for the binary system. It was obvious that a great deal of deviation was observed for the 10,14 and 12,14 quats (Figure 6), and also that some unpredicted phenomena must be active within the system.

Finally, to establish that the observed trends were not dependant on the oil used, we performed a series of tests using cyclohexane as opposed to decane. The same trends were observed, indicating 
that the relative data was dependent only upon the quat (Figure 7).

In conclusion, we have been able to demonstrate an effective means of evaluating surfactant at various concentrations. Furthermore, we have shown that there exist both synergistic and antagonistic factors within binary quat systems which are not predicted by the traditional model. This warrants further study into the interactions that occur within the binary system, and may lead to a better understanding of and improved formulations for biocides which contain quat compounds.

\section{ACKNOWLEDGEMENTS}

The authors are pleased to acknowledge the assistance of Southern Utah University and Albermarle Corporation in this research.

\section{REFERENCES}

1. www.infectioncontroltoday.com, accessed December 7, 2003.

2. A.N. Petrocci, in Disinfection, Sterilization and Preservation (S.S. Block, ed.), Lea \& Febiger, Philadelphia, 1983, pp. 309-329

3. J. N. Mbithi, V.S. Springthorpe, and S.A. Sattar, Appl. Environ, Microbiol. 56: 3601 (1990).

4. Miller, et al. US patent $5,599,990$, Oct. 27, 1995.

5. R. C. Larock, Comprehensive Organic Transformations, VCH Publishers, 1989, pp. 389-408.

\section{www.ajur.uni.edu}

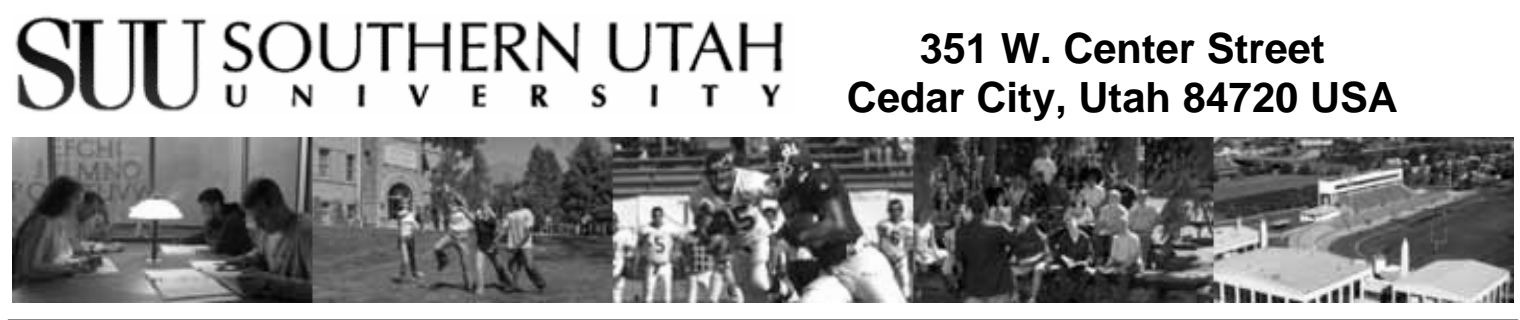

Through our more than 109 year history, Southern Utah University has evolved from a teacher training institution to its current role as a comprehensive, regional university offering graduate, baccalaureate, associate and technical programs. From the time of its founding, SUU has placed students first by featuring personalized and participative classes, combined with competent, qualified and supportive faculty, staff and administration. People of the region look to the University for skill development opportunities, major academic specialties, outreach services, cultural and athletic activities, economic and business development, and regional archives. National parks and recreation surround Cedar City. Skiing is $\mathbf{4 0}$ minutes away at Brian Head. For a wider range of metropolitan service, the city of Las Vegas is two-and-ahalf hours south, Provo two-and-a-half hours north.

Campus facilities vary from the historical Old Main and Braithwaite Liberal Arts Center building built in 1898 and 1899 to the Dixie Leavitt Business Building, to the Centrum special events center and classroom building, the Science Center, the Gerald R. Sherratt Library, the J.L. Sorenson Physical Education Building, and the new South Hall. As the home of the Utah Shakespearean Festival, SUU also features one of the world's most authentic Shakespearean stages and the Randall L. Jones Performing Arts Theatre. Guided tours of the University are available by appointment. Please contact the SUU Admissions Welcome Center at (435) 586-7740 to schedule tours, or visit http://www.suu.edu/you. 


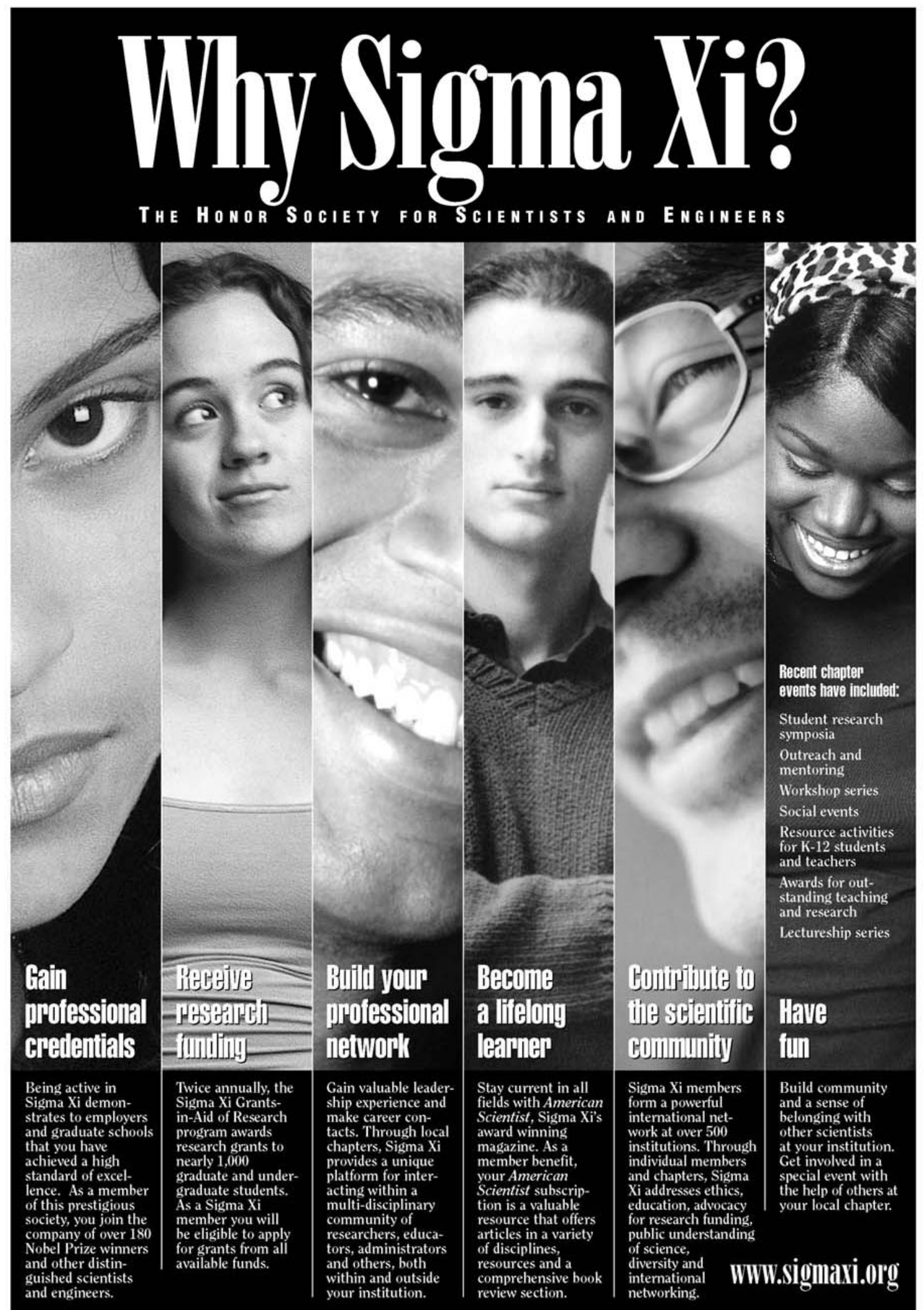

Sigma Xi, The Scientilic Research Society • 98 Alexanter Drive • P.O. Box 13975 • Research Triangle Park, NC 27708 • 818-549-4691 • 800-243-6534 